\title{
Exact Distributions of the Linear Combination of Gamma and Rayleigh Random Variables
}

\author{
Mohammad Shakil $^{1}$ and B. M. Golam Kibria ${ }^{2}$ \\ ${ }^{1}$ Miami Dade College, USA \\ ${ }^{2}$ Florida International University, USA
}

\begin{abstract}
The distribution of a linear combination of random variables arise in many applied problems, and have been extensively studied by different researchers. This article derived the exact distribution of the linear combination $a X+b Y$, where $a>0$ and $b$ are real constants, and $X$ and $Y$ denote gamma and Rayleigh random variables respectively and are distributed independently of each other. The associated cdfs and pdfs have been derived. The plots for the cdf and pdf, percentile points for selected coefficients and parameters, and the statistical application of the results have been provided. We hope the findings of the paper will be useful for practitioners in various fields.
\end{abstract}

Zusammenfassung: Die Verteilung einer Linearkombination von Zufallsvariablen tritt in vielen angewandten Problemen auf und wurden von verschiedenen Forscher umfangreich untersucht. In diesem Artikel wird die exakte Verteilung der Linearkombination $a X+b Y$ hergeleitet, wobei $a>0$ und $b$ reelle Konstanten sind, und $X$ und $Y$ eine Gamma- und Rayleigh $\mathrm{Zu}$ fallsvariable bezeichnen, welche unabhängig von einander verteilt sind. Die dazugehörenden cdfs und pdfs wurden hergeleitet. Abbildungen der cdf and pdf, Perzentile für ausgewählte Koeffizienten und Parameter, und die statistische Anwendung der Resultate werden bereitgestellt. Wir hoffen, dass die Ergebnisse in diesem Aufsatz brauchbar für Praktiker in verschiedenen Gebieten sein werden.

Keywords: Error Function, Hypergeometric Function, Incomplete Gamma Function, Parabolic Cylinder Function.

\section{Introduction}

The distributions of the linear combination of two independent random variables arise in many fields of research, see, for example, Ladekarl et al. (1997), Amari and Misra (1997), Cigizoglu and Bayazit (2000), Galambos and Simonelli (2005), Nadarajah and Kibria (2006a, 2006b), among others. In recent years, there has been a great interest in the study of the distributions of the linear combination $a X+b Y$, when $X$ and $Y$ are independent random variables and belong to different families, among them, Nadarajah and Kotz (2005) for the linear combination of exponential and gamma random variables, Kibria and Nadarajah (2007) for the linear combination of exponential and Rayleigh random variables, and Nason (2006) for the distributions of the sum $X+Y$, when $X$ and $Y$ are independent normal and sphered student's $t$ random variables respectively, are notable. This paper discusses the distributions of the linear combination $a X+b Y$, when 
$X$ and $Y$ are gamma and Rayleigh random variables and are distributed independently of each other.

The organization of this paper is as follows. In Section 2, the derivations of the cdfs and pdfs of linear combination $a X+b Y$ and corresponding plots have been provided. Percentage points of the new distribution for some selected parameters are given in Section 3. As an application, an example has been considered in Section 4. Some concluding remarks are provided in Section 5.

The derivations of the associated cdf's and pdf's in this paper involve some special functions, which are defined as follows. For details on these, see, for example, Abramowitz and Stegun (1970), Gradshteyn and Ryzhik (2000), Lebedev (1972), and Prudnikov et al. (1986), among others. The integrals $\Gamma(\alpha)=\int_{0}^{\infty} t^{\alpha-1} e^{-t} d t$, and $\gamma(\alpha, x)=$ $\int_{0}^{x} t^{\alpha-1} e^{-t} d t, \alpha>0$, are called (complete) gamma and incomplete gamma functions respectively, whereas the integral $\Gamma(\alpha, x)=\int_{x}^{\infty} t^{\alpha-1} e^{-t} d t, \alpha>0$, is called complementary incomplete gamma function. It is well-known that, for $\operatorname{Re}(p)>0$, and $\operatorname{Re}(\alpha)>0$, $\int_{0}^{\infty} x^{\alpha-1} e^{-p x} d x=\Gamma(\alpha) / p^{\alpha}$, and $\Gamma(\alpha, z)=\Gamma(\alpha)-\gamma(\alpha, z)=\int_{z}^{\infty} t^{\alpha-1} e^{-t} d t$. The functions defined by $\operatorname{erf}(x)=\frac{2}{\sqrt{\pi}} \int_{0}^{x} e^{-u^{2}} d u$, and $\operatorname{erfc}(x)=\frac{2}{\sqrt{\pi}} \int_{x}^{\infty} e^{-u^{2}} d u=1-\operatorname{erf}(x)$ are called error and complementary error functions respectively. The following series ${ }_{1} F_{1}(\alpha, \beta ; z)=\sum_{k=0}^{\infty} \frac{(\alpha)_{k} z^{k}}{(\beta)_{k} k !}$, (where $|z|<\infty, \beta \neq 0,-1,-2, \ldots$ ), is known as the confluent hypergeometric function of Kummer. The series

$$
\Psi(\alpha, \beta ; z)=\frac{\Gamma(1-\beta)}{\Gamma(\alpha-\beta+1)}{ }_{1} F_{1}(\alpha, \beta ; z)+\frac{\Gamma(\beta-1)}{\Gamma(\alpha)} z^{1-\beta}{ }_{1} F_{1}(\alpha-\beta+1,2-\beta ; z),
$$

(where $|z|<\infty, \beta \neq 0, \pm 1, \pm 2, \ldots$ ), is known as the confluent hypergeometric function of Tricomi. It is well-known that $\gamma(\alpha, z)=\left(z^{\alpha} / \alpha\right)_{1} F_{1}(\alpha, \alpha+1 ;-z)$, and $\Gamma(\alpha, z)=$ $e^{-z} \Psi(1-\alpha ; 1-\alpha ; z)$. The function $D_{p}(z)=\left(e^{-z^{2} / 4} / \Gamma(-p)\right) \int_{0}^{\infty} x^{-p-1} e^{-z x-x^{2} / 2} d x$, where $\operatorname{Re}(p)>0$. It is well-known that

$$
D_{p}(z)=2^{p / 2} e^{-z^{2} / 4} \Psi\left(-p / 2,1 / 2 ; z^{2} / 2\right), \quad D_{-1}(z)=\sqrt{\pi / 2} e^{z^{2} / 4} \operatorname{erfc}(z / \sqrt{2}),
$$

and $D_{-2}(z)=\sqrt{\pi / 2} e^{z^{2} / 4}\left\{\sqrt{2 / \pi} e^{-z^{2} / 2}-z \operatorname{erfc}(z / \sqrt{2})\right\}$ is called the parabolic-cylinder function.

\section{Distributions of the Linear Combination}

Let $Z=a X+b Y$, where $a>0$ and $b$ are real constants, and $X$ and $Y$ denote gamma and Rayleigh random variables respectively and are distributed independently of each other. In what follows, we consider the derivation of the distribution of the linear combination $Z=a X+b Y$. The gamma and Rayleigh random variables are defined as follows.

Gamma Distribution: A continuous random variable $X$ is said to have a gamma distribution if its pdf $f_{X}(x)$ and cdf $F_{X}(x)=P(X \leq x)$ are, respectively, given by

$$
f_{X}(x ; \alpha, \beta)=\frac{\beta^{\alpha}}{\Gamma(\alpha)} x^{\alpha-1} e^{-\beta x}, \quad x>0, \alpha>0, \beta>0
$$

and

$$
F_{X}(x)=\frac{\gamma(\alpha, \beta x)}{\Gamma(\alpha)}
$$


Rayleigh Distribution: A continuous random variable $Y$ is said to have a Rayleigh distribution if its pdf $f_{Y}(y)$ and cdf $F_{Y}(y)=P(Y \leq y)$ are, respectively, given by

$$
f_{Y}(y)=\left(y / \sigma^{2}\right) e^{-y^{2} / 2 \sigma^{2}}, \quad y>0, \sigma>0,
$$

and

$$
F_{Y}(y)=1-e^{-y^{2} / 2 \sigma^{2}}
$$

\subsection{Derivation of the CDF and PDF of $\mathrm{Z}=\mathrm{aX}+\mathrm{bY}$}

This section derives the expressions for the cdf and pdf of $Z=a X+b Y$. We can see that the distribution of the linear combination (LC) depends on the constants $a$ and $b$. Based on the conditions on $a$ and $b(a>0, b>0$ or $b<0)$, we have provided three different sets of CDF and PDF of the LC and provided them in Theorem 1.1, 1.2 and 1.3.

Theorem 1.1: Let $Z=a X+b Y$, where $X$ is a gamma random variable with pdf (1) and cdf (2), $Y$ is a Rayleigh random variable with pdf (3) and cdf (4), and $a$ and $b$ are real constants. Suppose, without loss of generality, that $a>0$. Then, if $b>0$ and $z>0$, the cdf and pdf of $Z$ are respectively given by

$$
\begin{aligned}
F_{Z}(z) & =\frac{\gamma\left(\alpha, \frac{\beta z}{a}\right)}{\Gamma(\alpha)}-\left[\frac{b \sigma}{\sqrt{2} \Gamma(\alpha)}\left(\frac{\beta}{a}\right)^{\alpha}\left(\frac{a z-\beta b^{2} \sigma^{2}}{a}\right)^{\alpha-1} e^{-\beta\left(2 a z-\beta b^{2} \sigma^{2}\right) / 2 a^{2}}\right. \\
& \left.\times \sum_{j=0}^{\infty} \frac{(-1)^{j} U^{j} \Gamma(\alpha)}{(j !) \Gamma(\alpha-j)}\left\{\gamma\left(\frac{j+1}{2}, \frac{\left(a z-\beta b^{2} \sigma^{2}\right)^{2}}{2 a^{2} b^{2} \sigma^{2}}\right)-\gamma\left(\frac{j+1}{2}, \frac{\beta^{2} b^{2} \sigma^{2}}{2 a^{2}}\right)\right\}\right]
\end{aligned}
$$

and

$$
\begin{aligned}
f_{Z}(z) & =\frac{1}{\Gamma(\alpha)}\left(\frac{\beta}{a}\right)^{\alpha} z^{\alpha-1} e^{-\beta z / a}-\frac{b \sigma}{\sqrt{2} \Gamma(\alpha)}\left(\frac{\beta}{a}\right)^{\alpha}\left(\frac{a z-\beta b^{2} \sigma^{2}}{a}\right)^{\alpha-1} e^{-\beta\left(2 a z-\beta b^{2} \sigma^{2}\right) / 2 a^{2}} \\
& \times \sum_{j=0}^{\infty} \frac{(-1)^{j} U^{j} \Gamma(\alpha)}{(j !) \Gamma(\alpha-j)}\left\{\frac{\beta}{a}\left[\gamma\left(\frac{j+1}{2}, \frac{\beta^{2} b^{2} \sigma^{2}}{2 a^{2}}\right)-\gamma\left(\frac{j+1}{2}, \frac{\left(a z-\beta b^{2} \sigma^{2}\right)^{2}}{2 a^{2} b^{2} \sigma^{2}}\right)\right]\right. \\
& +\frac{a(\alpha-j-1)}{\left(a z-\beta b^{2} \sigma^{2}\right)}\left[\gamma\left(\frac{j+1}{2}, \frac{\left(a z-\beta b^{2} \sigma^{2}\right)^{2}}{2 a^{2} b^{2} \sigma^{2}}\right)-\gamma\left(\frac{j+1}{2}, \frac{\beta^{2} b^{2} \sigma^{2}}{2 a^{2}}\right)\right] \\
& \left.+\frac{a z-\beta b^{2} \sigma^{2}}{a b^{2} \sigma^{2}}\left(\frac{a z-\beta b^{2} \sigma}{\sqrt{2} a b \sigma}\right)^{j-1} e^{-\left(a z-\beta b^{2} \sigma^{2}\right)^{2} /\left(2 a^{2} b^{2} \sigma^{2}\right)}\right\}
\end{aligned}
$$

where

$$
U=\frac{\sqrt{2} a b \sigma}{a z-\beta b^{2} \sigma^{2}}, \quad a>0, b>0, \alpha>0, \beta>0, \sigma>0, z>0 .
$$

Proof: Using expression (2) for the cdf of a gamma random variable $X$ and expression (3) for the pdf of a Rayleigh random variable $Y$, the cdf of the RV $Z=a X+b Y$ is given 
by

$$
\begin{aligned}
F_{Z}(z) & =\operatorname{Pr}(a X+b Y \leq z)=\operatorname{Pr}(X \leq(z-b Y) / a) \\
& =\int_{0}^{z / b} F_{X}\left(\frac{z-b y}{a}\right) f_{Y}(y) d y \\
& =\frac{1}{\sigma^{2} \Gamma(\alpha)} \int_{0}^{z / b} \gamma\left(\alpha, \frac{\beta(z-b y)}{a}\right) y e^{-y^{2} / 2 \sigma^{2}} d y \\
& =\gamma\left(\alpha, \frac{\beta z}{a}\right) / \Gamma(\alpha)-\left\{\frac{b}{\Gamma(\alpha)}\left(\frac{\beta}{a}\right)^{\alpha} e^{-\beta\left(2 a z-\beta b^{2} \sigma^{2}\right) / 2 a^{2}}\right. \\
& \left.\times \int_{0}^{z / b}(z-b y)^{\alpha-1} e^{-\frac{1}{\sqrt{2} \sigma}\left(y-\beta b \sigma^{2} / a\right)} d y\right\}
\end{aligned}
$$

The result for cdf in (5) easily follows by using the substitution $\left(y-\beta b \sigma^{2} / a\right) / \sqrt{2} \sigma=$ $t$, the binomial series representation $(1+w)^{k}=\sum_{j=0}^{\infty} \frac{\Gamma(k)}{\Gamma(k-j)} \frac{w^{j}}{j !}$, and the definition of the incomplete gamma function in (7). The pdf expression in (6) is easily obtained by differentiating the cdf in (5).

Theorem 1.2: Let $Z=a X+b Y$, where $X$ is a gamma random variable with pdf (1) and cdf (2), $Y$ is a Rayleigh random variable with pdf (3) and cdf (4), and $a$ and $b$ are real constants. Suppose, without loss of generality, that $a>0$. Then, if $b<0$ and $z \leq 0$, the cdf and pdf of $Z$ are respectively given by

$$
\begin{aligned}
F_{Z}(z) & =\left[\frac{b \sigma}{\sqrt{2} \Gamma(\alpha)}\left(\frac{\beta}{a}\right)^{\alpha}\left(\frac{a z-\beta b^{2} \sigma^{2}}{a}\right)^{\alpha-1} e^{-\beta\left(2 a z-\beta b^{2} \sigma^{2}\right) / 2 a^{2}}\right. \\
& \left.\times \sum_{j=0}^{\infty} \frac{(-1)^{j} U^{j} \Gamma(\alpha)}{(j !) \Gamma(\alpha-j)}\left\{\gamma\left(\frac{j+1}{2}, \frac{\left(a z-\beta b^{2} \sigma^{2}\right)^{2}}{2 a^{2} b^{2} \sigma^{2}}\right)-\Gamma\left(\frac{j+1}{2}\right)\right\}\right],
\end{aligned}
$$

and

$$
\begin{aligned}
f_{Z}(z)= & \frac{b \sigma}{\sqrt{2} \Gamma(\alpha)}\left(\frac{\beta}{a}\right)^{\alpha}\left(\frac{a z-\beta b^{2} \sigma^{2}}{a}\right)^{\alpha-1} e^{-\beta\left(2 a z-\beta b^{2} \sigma^{2}\right) / 2 a^{2}} \\
\times & \sum_{j=0}^{\infty} \frac{(-1)^{j} U^{j} \Gamma(\alpha)}{(j !) \Gamma(\alpha-j)} \\
& \left\{\frac{\beta}{a}\left[\gamma\left(\frac{j+1}{2}, \frac{\beta^{2} b^{2} \sigma^{2}}{2 a^{2}}\right)+\Gamma\left(\frac{j+1}{2}, \frac{\beta^{2} b^{2} \sigma^{2}}{2 a^{2}}\right)-\gamma\left(\frac{j+1}{2}, \frac{\left(a z-\beta b^{2} \sigma^{2}\right)^{2}}{2 a^{2} b^{2} \sigma^{2}}\right)\right]\right. \\
& +\frac{a(\alpha-j-1)}{a z-\beta b^{2} \sigma^{2}} \\
& {\left[\gamma\left(\frac{j+1}{2}, \frac{\left(a z-\beta b^{2} \sigma^{2}\right)^{2}}{2 a^{2} b^{2} \sigma^{2}}\right)-\gamma\left(\frac{j+1}{2}, \frac{\beta^{2} b^{2} \sigma^{2}}{2 a^{2}}\right)-\Gamma\left(\frac{j+1}{2}, \frac{\beta^{2} b^{2} \sigma^{2}}{2 a^{2}}\right)\right] } \\
& \left.+\frac{a z-\beta b^{2} \sigma^{2}}{a b^{2} \sigma^{2}}\left(\frac{a z-\beta b^{2} \sigma^{2}}{\sqrt{2} a b \sigma}\right)^{j-1} e^{-\left(a z-\beta b^{2} \sigma^{2}\right)^{2} /\left(2 a^{2} b^{2} \sigma^{2}\right)}\right\}
\end{aligned}
$$


where

$$
U=\frac{\sqrt{2} a b \sigma}{a z-\beta b^{2} \sigma^{2}}, \quad a>0, b>0, \alpha>0, \beta>0, \sigma>0, z>0 .
$$

Proof: Expressions (8) and (9) can be easily established similar to the Theorem 1.1.

Theorem 1.3: Let $Z=a X+b Y$, where $X$ is a gamma random variable with pdf (1) and cdf (2), $Y$ is a Rayleigh random variable with pdf (3) and cdf (4), and $a$ and $b$ are real constants. Suppose without loss of generality that $a>0$. Then, if $b<0$ and $z>0$, the cdf and pdf of $Z$ are respectively given by

$$
\begin{aligned}
F_{Z}(z) & =\gamma\left(\alpha, \frac{\beta z}{a}\right) / \Gamma(\alpha)-\left[\frac{b \sigma}{\sqrt{2} \Gamma(\alpha)}\left(\frac{\beta}{a}\right)^{\alpha}\left(\frac{a z-\beta b^{2} \sigma^{2}}{a}\right)^{\alpha-1} e^{-\beta\left(2 a z-\beta b^{2} \sigma^{2}\right) / 2 a^{2}}\right. \\
& \left.\times \sum_{j=0}^{\infty} \frac{(-1)^{j} U^{j} \Gamma(\alpha)}{(j !) \Gamma(\alpha-j)} \Gamma\left(\frac{j+1}{2}, \frac{\beta^{2} b^{2} \sigma^{2}}{2 a^{2}}\right)\right]
\end{aligned}
$$

and

$$
\begin{aligned}
f_{Z}(z) & =\frac{1}{\Gamma(\alpha)}\left(\frac{\beta}{a}\right)^{\alpha} z^{\alpha-1} e^{-\beta z / a}+\frac{b \sigma}{\sqrt{2} \Gamma(\alpha)}\left(\frac{\beta}{a}\right)^{\alpha}\left(\frac{a z-\beta b^{2} \sigma^{2}}{a}\right)^{\alpha-1} e^{-\beta\left(2 a z-\beta b^{2} \sigma^{2}\right) / 2 a^{2}} \\
& \times \sum_{j=0}^{\infty} \frac{(-1)^{j} U^{j} \Gamma(\alpha)}{(j !) \Gamma(\alpha-j)}\left(\frac{\beta}{a}-\frac{a(\alpha-j-1)}{a z-\beta b^{2} \sigma^{2}}\right) \Gamma\left(\frac{j+1}{2}, \frac{\beta^{2} b^{2} \sigma^{2}}{2 a^{2}}\right)
\end{aligned}
$$

where

$$
U=\frac{\sqrt{2} a b \sigma}{a z-\beta b^{2} \sigma^{2}}, \quad a>0, b>0, \alpha>0, \beta>0, \sigma>0, z>0 .
$$

Proof: Expressions (10) and (11) can be easily established similar to Theorem 1.1.

Corollary 1: Let $Z=a X+b Y$, where $X$ and $Y$ are distributed according to (1) and (3), respectively, and $a$ and $b$ are real constants. Suppose $\alpha=1$ in (1). Then, using the special properties of incomplete gamma and error functions, it is easy to see that the cdf and pdf of $Z$ can be expressed as follows:

(i) for $b>0, \beta>0, \sigma>0$, and $z>0$,

$$
F_{Z}(z)=1-e^{-\frac{\beta z}{a}}+\sqrt{\frac{\pi}{2}} \frac{\beta b \sigma}{a} e^{-\beta\left(2 a z-\beta b^{2} \sigma^{2}\right) / 2 a^{2}}\left\{\operatorname{erf}\left(\frac{\beta b \sigma}{\sqrt{2} a}\right)-\operatorname{erf}\left(\frac{\beta b^{2} \sigma^{2}-a z}{\sqrt{2} a b \sigma}\right)\right\}
$$

and

$$
\begin{aligned}
f_{Z}(z) & =\frac{\beta}{a}\left[e^{-\beta z / a}-e^{-z^{2} /\left(2 b^{2} \sigma^{2}\right)}+\left(\sqrt{\frac{\pi}{2}} \frac{\beta b \sigma}{a} e^{-\beta\left(2 a z-\beta b^{2} \sigma^{2}\right) / 2 a^{2}}\right)\right. \\
& \left.\times\left\{\operatorname{erf}\left(\frac{\beta b \sigma}{\sqrt{2} a}\right)-\operatorname{erf}\left(\frac{\beta b^{2} \sigma^{2}-a z}{\sqrt{2} a b \sigma}\right)\right\}\right]
\end{aligned}
$$


(ii) for $b<0, \beta>0, \sigma>0$, and $z \leq 0$,

$$
F_{Z}(z)=\sqrt{\frac{\pi}{2}} \frac{\beta b \sigma}{a} e^{-\beta\left(2 a z-\beta b^{2} \sigma^{2}\right) / 2 a^{2}}\left[\operatorname{erf}\left(\frac{\beta b^{2} \sigma^{2}-a z}{\sqrt{2} a b \sigma}\right)-1\right]
$$

and

$$
f_{Z}(z)=\frac{\beta}{a}\left[e^{-z^{2} /\left(2 b^{2} \sigma^{2}\right)}+\sqrt{\frac{\pi}{2}} \frac{\beta b \sigma}{a} e^{-\beta\left(2 a z-\beta b^{2} \sigma^{2}\right) / 2 a^{2}}\left\{1-\operatorname{erf}\left(\frac{\beta b^{2} \sigma^{2}-a z}{\sqrt{2} a b \sigma}\right)\right\}\right]
$$

(iii) for $b<0, \beta>0, \sigma>0$, and $z>0$,

$$
F_{Z}(z)=1-e^{-\frac{\beta z}{a}}\left[1+\sqrt{\frac{\pi}{2}} \frac{\beta b \sigma}{a} e^{\beta^{2} b^{2} \sigma^{2} / 2 a^{2}}\left\{1+\operatorname{erf}\left(\frac{\beta b \sigma}{\sqrt{2} a}\right)\right\}\right]
$$

and

$$
f_{Z}(z)=\frac{\beta}{a} e^{-\frac{\beta z}{a}}\left[1+\sqrt{\frac{\pi}{2}} \frac{\beta b \sigma}{a} e^{\beta^{2} b^{2} \sigma^{2} / 2 a^{2}}\left\{1+\operatorname{erf}\left(\frac{\beta b \sigma}{\sqrt{2} a}\right)\right\}\right] .
$$

The results in corollary 2.1 coincide with that of Kibria and Nadarajah (2007), where they considered the distribution of the linear combination of exponential and Rayleigh distribution.

Corollary 2: Let $Z=a X+b Y$, where $X$ and $Y$ are distributed according to (1) and (3), respectively, and $a$ and $b$ are real constants. Suppose $\alpha=1$ in (1). Then, from the case (i) of Corollary 1, that is, for $b>0, \beta>0, \sigma>0$, and $z>0$, it is easy to see that the cdf and pdf of $Z$ can be expressed in terms of parabolic-cylinder function $D_{p}(\cdot)$ and confluent hypergeometric function ${ }_{1} F_{1}(\cdot)$ as follows:

(I) CDF: for $b>0, \beta>0, \sigma>0$, and $z>0$,

(A)

$$
\begin{aligned}
F_{Z}(z) & =1-e^{-\beta z / a}-\frac{\beta \sigma^{2}}{a} e^{-\left(2 \beta a z-b^{2} \beta^{2} \sigma^{2}\right) / 2 a^{2}}\left[b^{2} \beta_{1} F_{1}\left(\frac{1}{2}, \frac{3}{2} ;-\frac{b^{2} \beta^{2} \sigma^{2}}{2 a^{2}}\right)\right. \\
& \left.-\frac{\beta b^{2} \sigma^{2}-a z}{\sigma^{2}}{ }_{1} F_{1}\left(\frac{1}{2}, \frac{3}{2} ;-\frac{\left(\beta b^{2} \sigma^{2}-a z\right)^{2}}{2 a^{2} b^{2} \sigma^{2}}\right)\right]
\end{aligned}
$$

(B)

$$
\begin{aligned}
F_{Z}(z) & =1-e^{-\beta z / a}-\frac{\sqrt{2} b \beta \sigma}{a} e^{-\left(2 \beta a z-b^{2} \beta^{2} \sigma^{2}\right) / 2 a^{2}} \\
& \times\left[e^{-\left(\beta b^{2} \sigma^{2}-a z\right)^{2} /\left(4 a^{2} b^{2} \sigma^{2}\right)} D_{-1}\left(\frac{\beta b^{2} \sigma^{2}-a z}{a b \sigma}\right)-e^{-\left(\beta^{2} b^{2} \sigma^{2} / 4 a^{2}\right.} D_{-1}\left(\frac{\beta b \sigma}{a}\right)\right]
\end{aligned}
$$

(II) PDF: for $b>0, \beta>0, \sigma>0$, and $z>0$,

(A)

$$
\begin{aligned}
f_{Z}(z) & =\frac{\beta}{a}\left[e^{-\beta z / a}-e^{-z^{2} /\left(2 b^{2} \sigma^{2}\right)}+\frac{b^{2} \beta^{2} \sigma^{2}}{a^{2}} e^{-\left(2 \beta a z-b^{2} \beta^{2} \sigma^{2}\right) / 2 a^{2}}\right. \\
& \left.\times\left\{\frac{\beta b^{2} \sigma^{2}-a z}{\beta b^{2} \sigma^{2}}{ }_{1} F_{1}\left(\frac{1}{2}, \frac{3}{2} ;-\frac{\left(\beta b^{2} \sigma^{2}-a z\right)^{2}}{2 a^{2} b^{2} \sigma^{2}}\right)-{ }_{1} F_{1}\left(\frac{1}{2}, \frac{3}{2} ;-\frac{b^{2} \beta^{2} \sigma^{2}}{2 a^{2}}\right)\right\}\right]
\end{aligned}
$$


(B)

$$
\begin{aligned}
f_{Z}(z) & =\frac{\beta}{a}\left[e^{-\beta z / a}-e^{-z^{2} /\left(2 b^{2} \sigma^{2}\right)}+\sqrt{\frac{\pi}{2}} \frac{b \beta \sigma}{a} e^{-\left(2 \beta a z-b^{2} \beta^{2} \sigma^{2}\right) / 2 a^{2}}\right. \\
& \left.\times\left\{e^{-\left(\beta b^{2} \sigma^{2}-a z\right)^{2} /\left(4 a^{2} b^{2} \sigma^{2}\right)} D_{-1}\left(\frac{\beta b^{2} \sigma^{2}-a z}{a b \sigma}\right)-e^{-\beta^{2} b^{2} \sigma^{2} / 4 a^{2}} D_{-1}\left(\frac{\beta b \sigma}{a}\right)\right\}\right]
\end{aligned}
$$

Remark: Using the definition of parabolic-cylinder function $D_{p}(\cdot)$ and confluent hypergeometric function ${ }_{1} F_{1}(\cdot)$, as discussed in Section 1 , one can easily obtain the expressions for the cdf and pdf of $Z=a X+b Y$ in terms of these special functions for cases (ii) and (iii) of Corollary 1.

Corollary 3: Suppose $X$ and $Y$ are distributed according to (1) and (3), respectively. Suppose $\alpha=1$ in (1). Then, one can easily obtain the cdf and pdf of $Z=X+Y$ from (i) in Corollary 1 as

$$
F_{Z}(z)=1-e^{-\beta z}+\sqrt{\frac{\pi}{2}} \beta \sigma e^{-\beta\left(2 z-\beta \sigma^{2}\right) / 2}\left\{\operatorname{erf}\left(\frac{\beta \sigma}{\sqrt{2}}\right)-\operatorname{erf}\left(\frac{\beta \sigma^{2}-z}{\sqrt{2} \sigma}\right)\right\}
$$

and

$f_{Z}(z)=\beta\left[e^{-\beta z}-e^{-z^{2} / 2 \sigma^{2}}+\sqrt{\frac{\pi}{2}} \beta \sigma e^{-\beta\left(2 z-\beta \sigma^{2}\right) / 2}\left\{\operatorname{erf}\left(\frac{\beta \sigma}{\sqrt{2}}\right)-\operatorname{erf}\left(\frac{\beta \sigma 2-a z}{\sqrt{2} \sigma}\right)\right\}\right]$,

where $\beta>0, \sigma>0$, and $z>0$.

Corollary 4: Suppose $X$ and $Y$ are distributed according to (1) and (3), respectively. Suppose $\alpha=1$ in (1). Then, one can easily obtain the cdf and pdf of $Z=X-Y$ from (iii) in Corollary 1 as

$$
F_{Z}(z)=1-e^{-\beta z}\left[1+\sqrt{\frac{\pi}{2}} \beta \sigma e^{\beta^{2} \sigma^{2} / 2}\left\{1+\operatorname{erf}\left(\frac{\beta \sigma}{\sqrt{2}}\right)\right\}\right]
$$

and

$$
f_{Z}(z)=\beta e^{-\beta z}\left[1+\sqrt{\frac{\pi}{2}} \beta \sigma e^{\beta^{2} \sigma^{2} / 2}\left\{1+\operatorname{erf}\left(\frac{\beta \sigma}{\sqrt{2}}\right)\right\}\right],
$$

where $\beta>0, \sigma>0$, and $z>0$.

\subsection{Plots of the CDF and PDF of $\mathrm{Z}=\mathrm{aX}+\mathrm{bY}$}

Using Maple 11, the possible shapes of the cdfs and pdfs of $Z=a X+b Y$ are provided for some selected values of coefficients and parameters in Figures 1 to 4 below. The effects of the parameters can easily be seen from these graphs.

From these graphs, it is evident that the distribution of $z$ is right skewed. Similar plots can be drawn for others values of the parameters. 

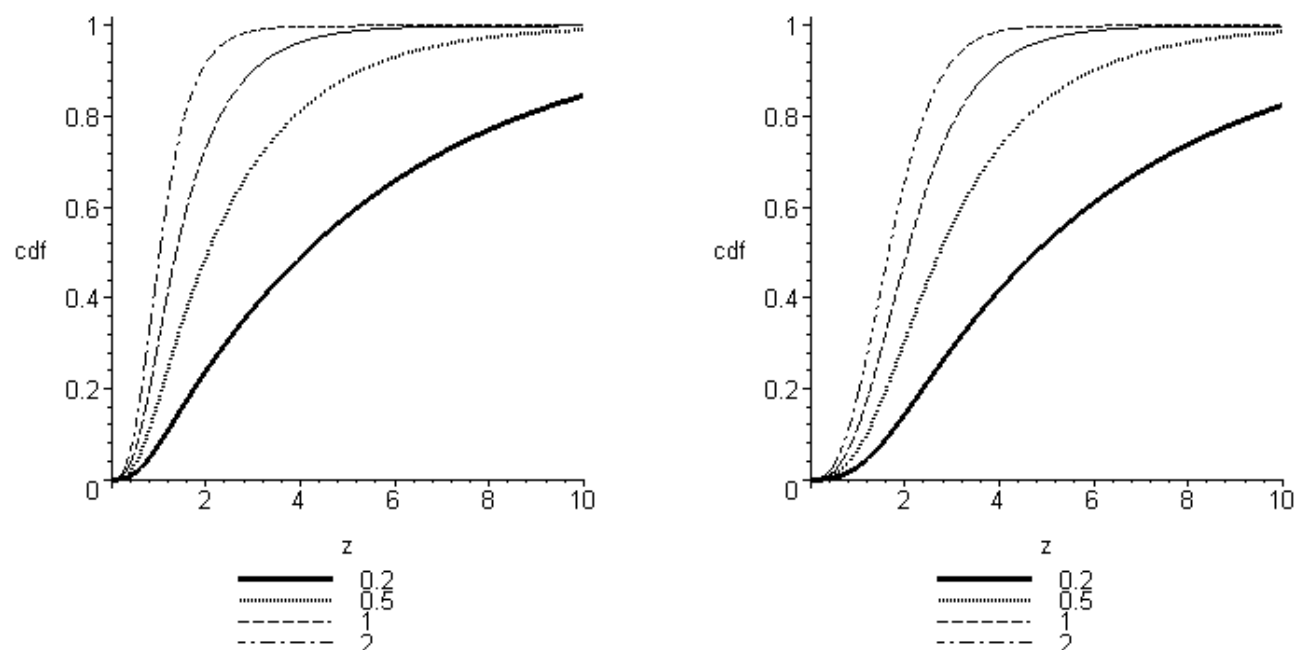

Figure 1: CDF plots of $Z=a X+b Y$ for $a=1, b=1, \alpha=1, \sigma=0.5, \beta=0.2,0.5,1,2$ (left), and $a=1, b=1, \alpha=1, \sigma=1, \beta=0.2,0.5,1,2$ (right).
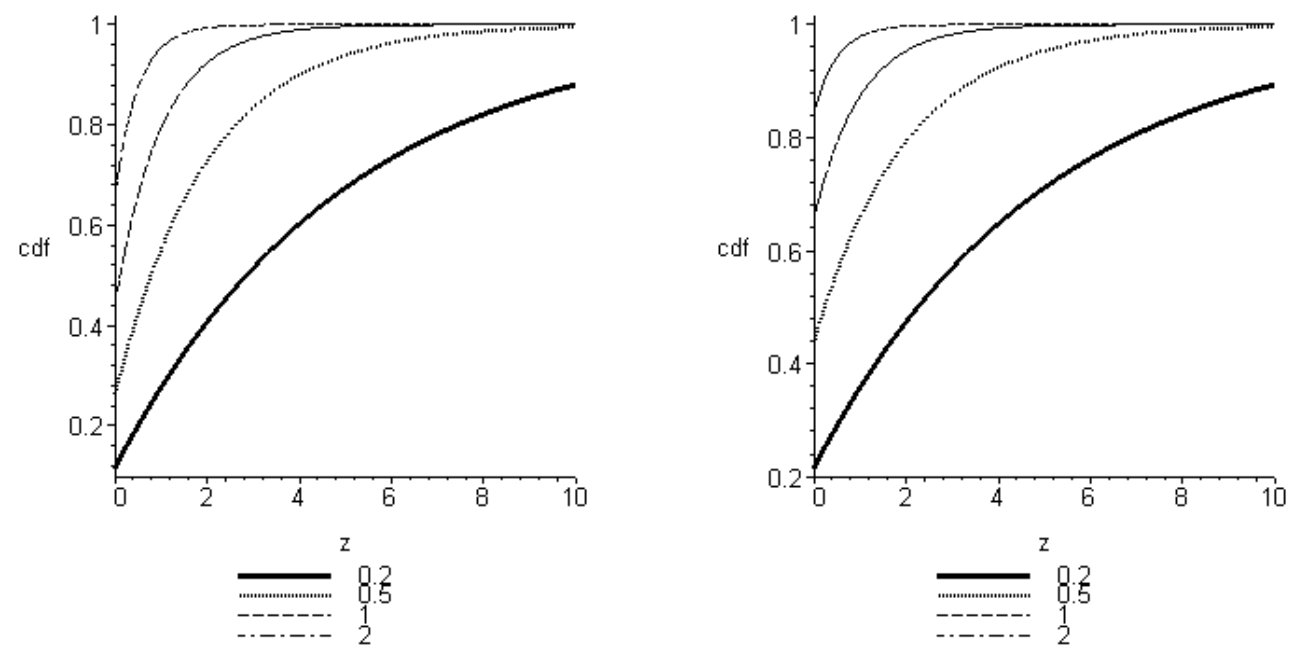

Figure 2: CDF plots of $Z=a X+b Y$ for $a=1, b=-1, \alpha=1, \sigma=0.5, \beta=$ $0.2,0.5,1,2$ (left), and $a=1, b=-1, \alpha=1, \sigma=1, \beta=0.2,0.5,1,2$ (right).
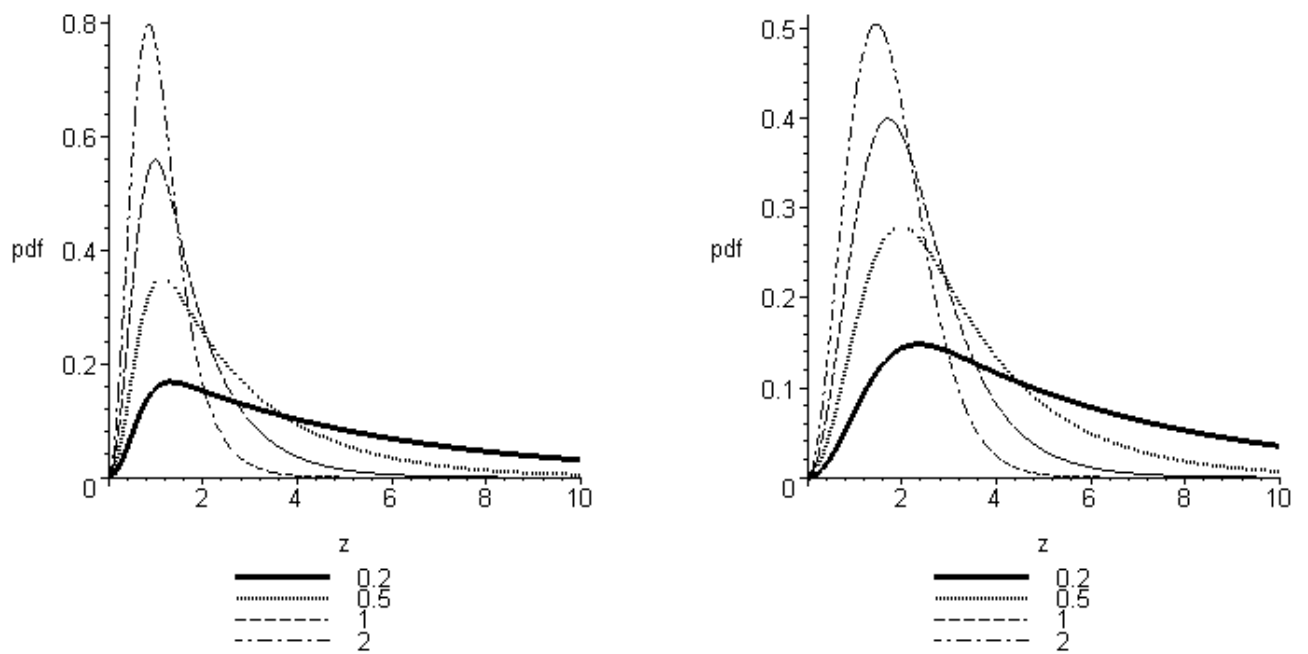

Figure 3: PDF plots of $Z=a X+b Y$ for $a=1, b=1, \alpha=1, \sigma=0.5, \beta=0.2,0.5,1,2$ (left), and $a=1, b=1, \alpha=1, \sigma=1, \beta=0.2,0.5,1,2$ (right). 

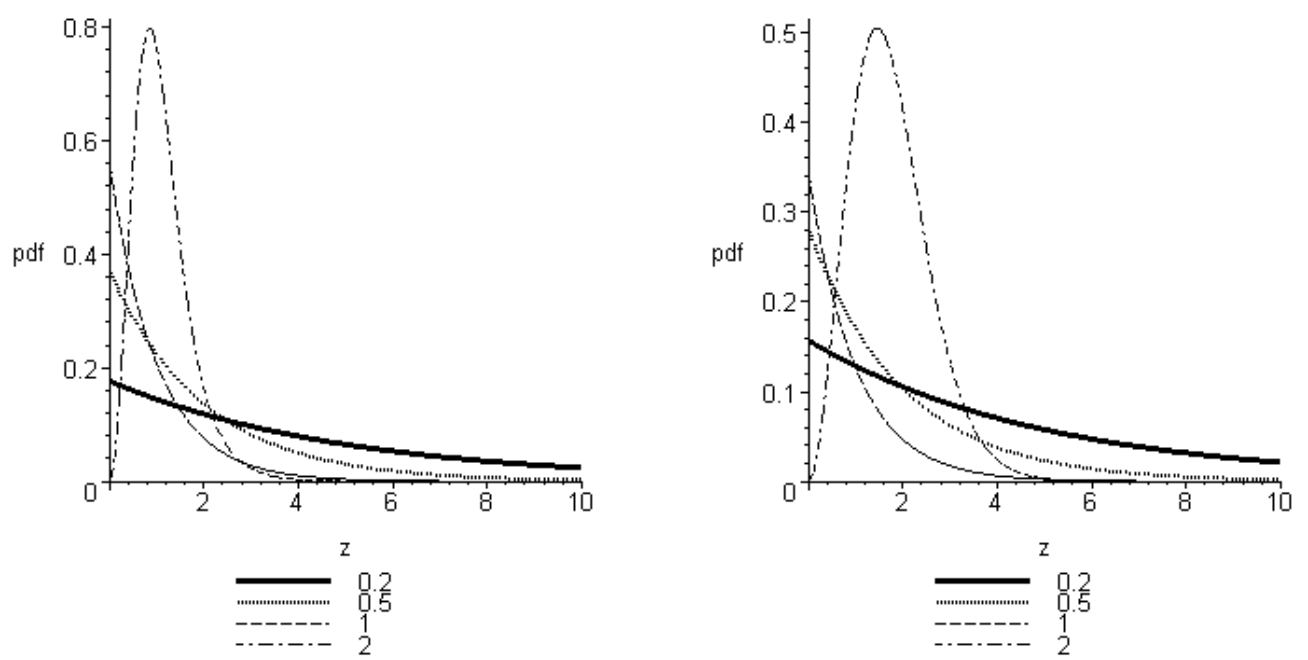

Figure 4: PDF plots of $Z=a X+b Y$ for $a=1, b=-1, \alpha=1, \sigma=0.5, \beta=0.2,0.5,1,2$ (left), and $a=1, b=-1, \alpha=1, \sigma=1, \beta=0.2,0.5,1,2$ (right).

\section{Percentiles}

This section computes the percentage points of the distribution of the LC $Z=a X+b Y$, where $a>0$ and $b$ are real constants. For any $0<p<1$, the $100 p$ th percentile (also called the quantile of order $p$ ) of the distribution with pdf $f_{Z}(z)$ is a number $z_{p}$ such that the area under $f_{Z}(z)$ to the left of $z_{p}$ is $p$. That is, $z_{p}$ is any root of the equation

$$
F\left(z_{p}\right)=\int_{-\infty}^{z_{p}} f_{Z}(u) d u=p
$$

By numerically solving equation (12) for the cdf in Theorem 1.1 using a Maple 11 program, percentage points $z_{p}$ associated with the cdf of $Z$ are computed for some selected values of the parameters. These are provided in Table 1 and 2 .

\section{Applications}

To illustrate the performance of the new distribution, an example of rain fall data has been considered in this section. We have considered the maximum 24-hour precipitation recorded for thirty-six inland hurricanes (1900-1969) (Source: Larsen and Marx, 2006, p. 360. The mean, median and skewness of this data are 7.29, 5.58 and 2.52, respectively. We can see that the data are right skewed. The rain-fall data is provided in Table 3.

Maple 11 has been used for computing the data moments, estimating the parameter (by the method of moments), and chi-square test for goodness-of-fit. The data moments are computed as $\hat{\mu}_{1}=7.29, \hat{\mu}_{2}=85.59$ and $\hat{\mu}_{3}=1543.64$. The estimation of the parameters and chi-square goodness-of-fit test are provided in Table 4 and 5, respectively. From the chi-square goodness-of-fit test we observed that the gamma, Rayleigh and LC model fit the rainfall data reasonably well. However, the LC model produces the highest p-value and therefore fitted better than the gamma and Rayleigh distribution. Also, for the parameters estimated in Table 4, gamma, Rayleigh, and the LC model have been 
Table 1: Percentage points of $Z=X+Y$ for $\alpha=1$.

\begin{tabular}{cccccccc}
\hline$\sigma$ & $\beta$ & $75 \%$ & $80 \%$ & $85 \%$ & $90 \%$ & $95 \%$ & $99 \%$ \\
\hline 0.5 & 0.2 & 3.626579 & 3.736235 & 3.843157 & 3.947593 & 4.049755 & 4.129971 \\
& 0.5 & 2.632961 & 2.711655 & 2.788662 & 2.864144 & 2.938239 & 2.996599 \\
& 1.0 & 2.144516 & 2.209016 & 2.272378 & 2.334725 & 2.396162 & 2.444716 \\
& 2.0 & 1.817671 & 1.873934 & 1.929467 & 1.984368 & 2.038720 & 2.081854 \\
1.0 & 0.2 & 4.211928 & 4.323557 & 4.432266 & 4.538329 & 4.641980 & 4.723301 \\
& 0.5 & 3.205419 & 3.287408 & 3.367389 & 3.445570 & 3.522126 & 3.582302 \\
& 1.0 & 2.708586 & 2.777780 & 2.845389 & 2.911590 & 2.976534 & 3.027672 \\
& 2.0 & 2.377645 & 2.440037 & 2.501121 & 2.561056 & 2.619977 & 2.666463 \\
\hline
\end{tabular}

Table 2: Percentage points of $Z=X-Y$ for $\alpha=1$.

\begin{tabular}{cccccccc}
\hline$\sigma$ & $\beta$ & $75 \%$ & $80 \%$ & $85 \%$ & $90 \%$ & $95 \%$ & $99 \%$ \\
\hline 0.5 & 0.2 & 2.476823 & 2.583890 & 2.688448 & 2.790727 & 2.890906 & 2.969647 \\
& 0.5 & 1.548095 & 1.623300 & 1.697144 & 1.769743 & 1.841200 & 1.897604 \\
& 1.0 & 1.130406 & 1.191086 & 1.251018 & 1.310269 & 1.368899 & 1.415391 \\
& 2.0 & 0.893320 & 0.946215 & 0.998805 & 1.051126 & 1.103206 & 1.144716 \\
1.0 & 0.2 & 2.091253 & 2.193567 & 2.293780 & 2.392052 & 2.488529 & 2.564502 \\
& 0.5 & 1.281635 & 1.352142 & 1.421667 & 1.490283 & 1.558052 & 1.611698 \\
& 1.0 & 0.962859 & 1.020183 & 1.077045 & 1.133480 & 1.189521 & 1.234089 \\
& 2.0 & 0.813168 & 0.864682 & 1.077045 & 0.967269 & 1.018373 & 1.059177 \\
\hline
\end{tabular}

Table 3: Rainfall data (see Larsen and Marx, 2006).

\begin{tabular}{rrrrrrrrr}
\hline 31.00 & 2.82 & 3.98 & 4.02 & 9.50 & 4.50 & 11.40 & 10.71 & 6.31 \\
4.95 & 5.64 & 5.51 & 13.40 & 9.72 & 6.47 & 10.16 & 4.21 & 11.60 \\
4.75 & 6.85 & 6.25 & 3.42 & 11.80 & 0.80 & 3.69 & 3.10 & 22.22 \\
7.43 & 5.00 & 4.58 & 4.46 & 8.00 & 3.73 & 3.50 & 6.20 & 0.67 \\
\hline
\end{tabular}

Table 4: Parameter estimates for the rainfall data assuming different models.

\begin{tabular}{cccc}
\hline \multicolumn{4}{c}{ Model } \\
& LC $Z=X+Y$ & Gamma & Rayleigh \\
\hline$\hat{\alpha}$ & 1 & 1.63500 & \\
$\hat{\beta}$ & 0.17751 & 0.22436 & \\
$\hat{\sigma}$ & 1.31986 & & 5.8146 \\
\hline
\end{tabular}

Table 5: Comparison criteria (chi-square test for goodness-of-fit).

\begin{tabular}{lccc}
\hline & \multicolumn{3}{c}{ Model } \\
& LC $Z=X+Y$ & Gamma & Rayleigh \\
\hline Test statistic & 0.600 & 0.759 & 1.050 \\
Critical value & 5.991 & 5.991 & 5.991 \\
p-value & 0.740 & 0.684 & 0.591 \\
\hline
\end{tabular}

superimposed on the histogram of the rainfall data as in Figure 5, from which we observed that the LC fits the rainfall data reasonably well. 


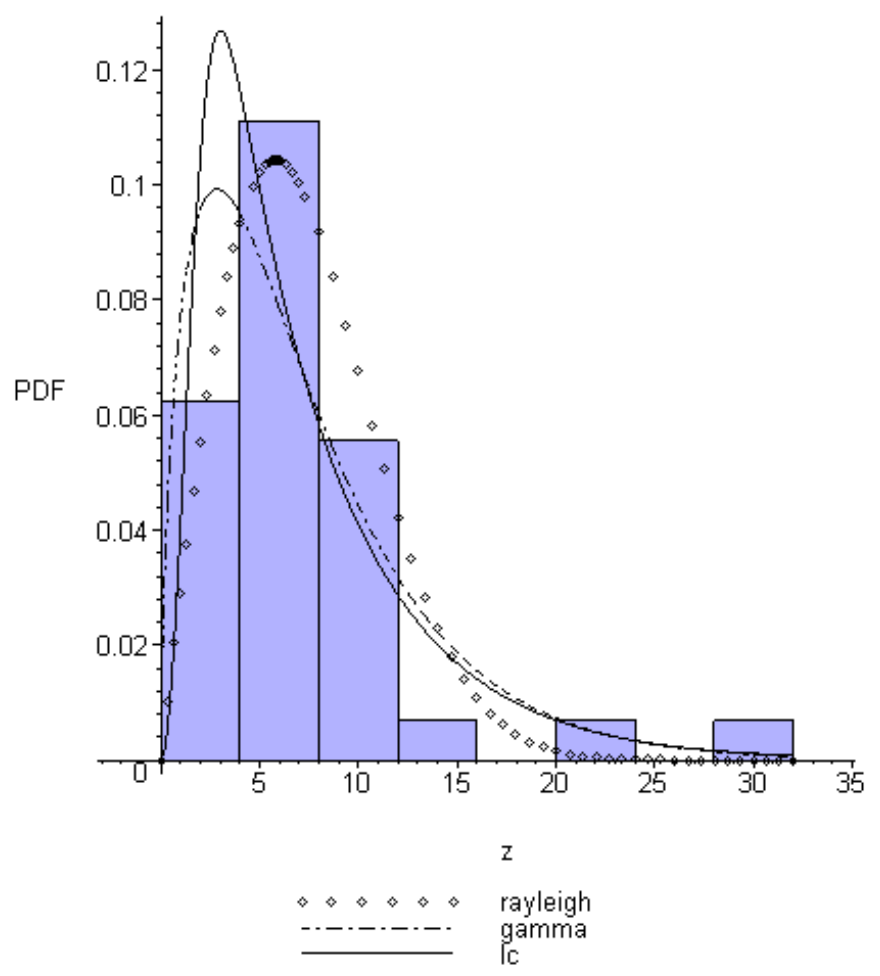

Figure 5: Fitting the pdfs under the LC, gamma and Rayleigh model for the rainfall data.

\section{Concluding Remarks}

This paper has derived the exact probability distribution of the linear combination of two independent random variables $X$ and $Y$, where $X$ has a Rayleigh and $Y$ has a gamma distribution. The expressions for cdf and pdf of the linear combination of two independent random variables are given. The plots for the cdf and pdf, percentile points for selected coefficients and parameters, and the statistical application of the distributions have been provided. We considered the rainfall data and found that LC fitted better than both gamma and Rayleigh distributions. Note that Kibria and Nadarajah (2007) considered the linear combination of exponential and Rayleigh distributions and therefore, Kibria and Nadarajah (2007) is a special case of this paper. We hope the findings of the paper will be useful for the practitioners and encourage the young scientists to do more researches in these topics.

\section{Acknowledgments}

The authors would like to thank the editor and referee for their useful comments and suggestions which considerably improved the presentation of the paper. 


\title{
References
}

Abramowitz, M., and Stegun, I. A. (1970). Handbook of Mathematical Functions with Formulas, Graphs, and Mathematical Tables. New York: Dover.

Amari, S. V., and Misra, R. B. (1997). Closed-form expressions for distribution of sum of exponential random variables. IEEE Transactions on Reliability, 46, 519-522.

Cigizoglu, H. K., and Bayazit, M. (2000). A generalized seasonal model for flow duration curve. Hydrological Processes, 14, 1053-1067.

Galambos, J., and Simonelli, I. (2005). Products of Random Variables - Applications to Problems of Physics and to Arithmetical Functions. Boca Raton: CRC Press.

Gradshteyn, I. S., and Ryzhik, I. M. (2000). Table of Integrals, Series, and Products (6th ed.). San Diego: Academic Press.

Kibria, B. M. G., and Nadarajah, S. (2007). Reliability modeling: Linear combination and ratio of exponential and Rayleigh. IEEE Transactions on Reliability, 56, 102-105.

Ladekarl, M., Jensen, V., and Nielsen, B. (1997). Total number of cancer cell nuclei and mitoses in breast tumors estimated by the optical disector. Analytical and Quantitative Cytology and Histology, 19, 329-337.

Larsen, R. J., and Marx, M. L. (2006). An Introduction to Mathematical Statistics and its Applications (4th ed.). New Jersey: Pearson-Prentice Hall.

Lebedev, N. N. (1972). Special Functions and their Applications. New York: Dover.

Nadarajah, S., and Kibria, B. M. G. (2006a). On the ratio of generalized Pareto random variables. Stochastic Environmental Research \& Risk Assessment, 206-212.

Nadarajah, S., and Kibria, B. M. G. (2006b). Drought models based on Burr XII variables. Applications Mathematicae, 33, 185-193.

Nadarajah, S., and Kotz, S. (2005). On the linear combination of exponential and gamma random variables. Entropy, 7, 161-171.

Nason, G. (2006). On the sum of $\mathrm{t}$ and Gaussian random variables. Statistics and Probability Letters, 76, 1280-1286.

Prudnikov, A. P., Brychkov, Y. A., and Marichev, O. I. (1986). Integrals and Series (Volumes 1, 2, and 3). Amsterdam: Gordon and Breach Science Publishers.

\author{
Authors' Addresses \\ Mohammad Shakil \\ Department of Mathematics \\ Miami Dade College, Hialeah Campus \\ 1780 West 49th Street \\ Hialeah, FL 33012, USA \\ E-mail: mshakil@mdc.edu \\ B. M. Golam Kibria \\ Department of Statistics \\ Florida International University \\ University Park \\ Miami, Florida 33199, USA \\ E-mail: kibriag@fiu.edu
}

